\section{Weniger Nutzen von Radiochemotherapie}

\begin{abstract}
Ältere Patienten mit nichtkleinzelligem Lungenkarzinom (NSCLC) des Stadiums III profitieren weniger als jüngere von der simultanen Strahlenchemotherapie. Das ergab die Analyse von Patientendaten aus 16 Studien der Phase II oder III, die zwischen 1990 und 2012 mit Unterstützung des amerikanischen National Cancer Institute durchgeführt worden waren. In allen Studien hatten Patienten mit einem NSCLC des Stadiums III eine Radiochemotherapie allein oder ergänzt um eine Konsolidierungs- oder Induktionschemotherapie erhalten.

Das Gesamtüberleben älterer Patienten war gegenüber jüngeren schlechter. In der adjustierten Analyse ergab sich eine $\mathrm{Ha}$ zard Ratio (HR) von 1,17. Dagegen war das progressionsfreie Überleben in beiden Altersgruppen vergleichbar (HR 1,00).
\end{abstract}

Unerwünschte Ereignisse des Grads $\geq 3$ waren bei älteren Patienten häufiger (Odds Ratio [OR] 1,38). Unerwünschte Ereignis-

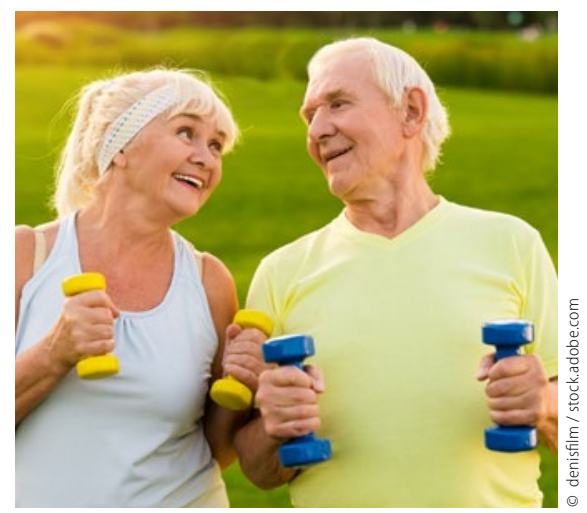

An der Studie nahmen eher fitte Senioren teil, trotzdem lebten sie weniger lang als Jüngere. se vom Grad 5 mussten bei Älteren mehr als doppelt so häufig verzeichnet werden wie bei Jüngeren ( 9 vs. $4 \%$; $<<0,01$ ).

Ein vergleichbares progressionsfreies Überleben, aber eine erhöhte Zahl von unerwünschten Ereignisse vom Grad 5 interpretieren die Forscher als Hinweise auf eine Rolle der Frühmortalität bei schlechterem Gesamtüberleben der Älteren. Diese erhielten zudem die Therapie weniger häufig vollständig als jüngere (47 vs. 57\%; $\mathrm{p}<0,01)$, brachen die Therapie häufiger wegen unerwünschter Ereignisse ab (20 vs. $13 \%$; $<0,01)$, starben noch während der Therapie ( 7,8 vs. $2,9 \%$; $p<0,01)$ und verweigerten häufiger eine weitere Therapie $(5,8$ vs. $3,9 \% ; p=0,02)$ Friederike Klein

Stinchcombe TE et al. Pooled Analysis of Individual Patient Data on Concurrent Chemoradiotherapy for Stage III Non-Small-Cell Lung Cancer in Elderly Patients Compared With Younger Patients Who Participated in US National Cancer Institute Cooperative Group Studies. J Clin Oncol. 2017; 35(25):2885-92.

\section{Bessere prognostische Einschätzung}

An einer großen Patientengruppe mit multiplem Myelom wurde der revidierte Myeloma Comorbidity Index (R-MCI) entwickelt und validiert. In die zusammenfassende Evaluation von Komorbidität, Gebrechlichkeit und Funktionseinschränkungen gingen die Daten von 801 konsekutiven Patienten ein. Eine multivariate Analyse zeigte, dass Nieren- und Lungenfunktion, der Karnofsky-Performancestatus, Gebrechlichkeit und Alter signifikante Risikofaktoren für ein schlechteres Gesamtüberleben sind. Andere mutmaßliche Risiken wie Leberoder gastrointestinale Störungen erreichten keine Signifikanz.

Die bestätigten fünf Faktoren mit einem maximalen Score von neun Punkten wurden in den gewichteten revidierten Myeloma Comorbidity Index (R-MCI) aufgenommen, der eine Einteilung der Patienten in drei Gruppen erlaubt: fitte Patienten R-MCI $\leq 3$ ( $\mathrm{n}=247 ; 30,8 \%$ ), intermediär-fitte Patienten R-MCI 4-6 ( $\mathrm{n}=$ 446; 55,7\%) und gebrechliche Patienten R-MCI > 6 ( $n=108 ; 13,5 \%)$. Diese Subgruppen wurden durch die Validierungs- analyse bestätigt und zeigten mediane Gesamtüberlebensraten von 10,1 Jahren, 4,4 Jahren und 1,2 Jahren.

Der R-MCI wurde mit dem Charlson Comorbidity Index, dem Hematopoietic Cell Transplantation-Specific Comorbidity Index sowie dem Kaplan-Feinstein Index verglichen. Wenn man diese ebenfalls in Risikogruppen, basierend auf den $25 \%$ - und $75 \%$-Quartilen unterteilte, zeigten sich die höchsten Hazard Ratios, die besten Voraussagegenauigkeiten und die besten Brier-Scores für den R-MCI.

Ein entscheidender Vorteil des R-MCI ist, dass seine Angaben in der Regel leicht der Anamnese und der Krankenakte entnommen werden können. Brigitte Schalhorn

Engelhardt $\mathrm{M}$ et al. A concise revised myeloma comorbidity index as a valid prognostic instrument in a large cohort of 801 multiple myeloma patients. Haematologica. 2017;102(5):2-13.

\section{kurz notiert}

\section{Zika-Virus killt Tumorstammzellen im Gehirn}

Im Gehirn von Ungeborenen können Zika-Viren schwere Schäden anrichten, indem sie gezielt neurale Vorläuferzellen, aus denen neue Nervenzellen entstehen, infizieren und abtöten. Diese tödliche Kraft der Viren konnten amerikanische Forscher ausnutzen und gegen maligne Zellen im Gehirn einsetzen. In Glioblastomgewebe konnten sie zeigen, dass das Zika-Virus Tumorstammzellen abtötet. Tumorstammzellen überleben häufig Chemotherapie und Bestrahlung und produzieren weiterhin Tumorzellen. Eine Infektion des Gehirns mit Zika-Viren und die Radiochemotherapie könnten somit komplementäre Effekte haben. Die Standardtherapie tötet die Hauptmasse der Tumorzellen, lässt aber meist intakte Stammzellen zurück. Das Zika-Virus greift dagegen die Stammzellen an, lässt aber andere Tumorzellen am Leben. Im Mausmodell führte die Injektion von Zika-Viren zu einer deutlichen Reduktion von Hirntumoren. Die infizierten Mäuse lebten außerdem signifikant länger. Bei Erwachsenen sind Zika-Viren weniger gefährlich, weil sie im Gegensatz zu Kindern nur selten Vorläuferzellen aufweisen. In Untersuchungen an Epilepsiepatienten konnten die Forscher bereits nachweisen, dass Zika-Viren keine Nichttumorzellen infizieren [Zhu Z et al. J Exp Med. 2017; Sep 5 (Epub ahead of print)]. Judith Neumaier 